\title{
Os atributos afetivos para a docência na graduação a distância ${ }^{1}$
}

Cláudio Rodrigues da Cunha - Universidade Federal do Rio Grande do Sul crcunha@gmail.com

Sérgio Roberto Kieling Franco - Universidade Federal do Rio Grande do Sul sergio.franco@ufrgs.br

Magda Bercht - Universidade Federal do Rio Grande do Sul - bercht@inf.ufrgs.br

Resumo. Apresentam-se os principais atributos afetivos dos docentes em graduações a distância a partir de pesquisa de campo em universidades do Rio Grande do Sul, associadas a experiências em psicoterapia de grupos desenvolvidas por Irving Yalom, mantendo as discussões num âmbito interdisciplinar. Buscou-se a classificação e a identificação dos atributos afetivos, como organização, comunicabilidade e dedicação, visando sistematizar uma formação afetiva dos professores. Os resultados obtidos mostram a possibilidade do desenvolvimento de novas estratégias relacionais $e$ instrumentos didáticos na formação e acompanhamento dos docentes naquela modalidade de ensino.

Palavras-chave. Atributos afetivos. graduação a distância. formação de professores.

\section{The Affective attributes to teaching degrees in distance education}

\begin{abstract}
This article aims to show which are the main affective attributes needed to teaching degrees who work in distance education universities in the State of Rio Grande do Sul, associated to psychotherapy group experiences developed by Irving Yalom, highlighting the discussion within an interdisciplinary context. The applied questions sought to apply the classification and identification of the major teacher affective features, as organization, communicability and dedication, to systematize their affective training. The results show that there is a possibility of developing new relational, didactic training and mentoring strategies for teachers in that modality of education.
\end{abstract}

Keywords. Affective attributes. undergraduate distance. teacher education.

\footnotetext{
${ }^{1}$ Artigo elaborado a partir da Tese de Doutorado, com o mesmo título, de Cláudio Rodrigues da Cunha, defendida em 16 de agosto de 2012, no Programa de Pós Graduação em Informática na Educação da Universidade Federal do Rio Grande do Sul.
} 


\section{Introdução}

Considerando que a Educação a Distância (EAD) oferece uma nova perspectiva de ensino, onde a ausência do contato físico direto parece compor um de seus obstáculos mais ansiogênicos (Cunha et al, 2006), torna-se necessário discutir e apresentar novas sistematizações que minimizem tais dificuldades a partir do aprimoramento das capacidades afetivas dos envolvidos que possam influenciar a atuação docente.

Nesse sentido, o presente artigo utilizou conhecimentos e experiências de áreas pontuais da Psicoterapia de Grupos (com enfoque nos trabalhos do psicanalista existencial Irving Yalom) para identificar as dimensões afetivas mais importantes ao professor que trabalha em graduação a distância.

Investigou-se de que forma ocorrem as afetações docentes em cursos mediados pelo computador, a partir de entrevistas com atores do processo de ensino, para identificar os principais atributos afetivos realizando um estudo de casos múltiplos junto a uma amostra em cursos de graduação em EAD no Rio Grande do Sul, oferecendo recomendações não só para aqueles que organizam atividades de formação continuada docente, como também para os próprios interessados que desejarem buscar um auto aperfeiçoamento em suas práticas educacionais.

Espera-se que a investigação ofereça um melhor suporte aos trabalhos educacionais à distância para uma melhora contínua das práticas profissionais dos sujeitos pesquisados.

\section{Motivação}

Os principais responsáveis na condução dos procedimentos afetivos de uma sala de aula são os professores, pois se espera deles a maturidade e a experiência no papel de condutores de suas turmas (que lidam com alunos de diferentes tipos de personalidades, diferentes disponibilidades de tempo e que representam diversas parcelas da sociedade).

Entretanto, suas características afetivas acabam não sendo consideradas objetivamente na concepção e utilização dos ambientes virtuais. Em pesquisas anteriores (BRASIL, 2001), verificou-se que os atributos afetivos de alunos podem ser desenvolvidos e avaliados. A delimitação, a definição e a forma de desenvolvê-los devem ser decididas em comum acordo entre os professores e seus respectivos coordenadores, levando em consideração as necessidades relacionais de cada grupo.

Voltando o foco para os professores, um docente preparado afetivamente (de acordo com os conteúdos a serem ministrados, as características de sua turma, o ambiente físico disponível, os apoios de suas coordenações e tutores, a cultura da instituição onde trabalha e o momento social que todos vivenciam), terá melhores condições de desenvolver as atividades planejadas e alcançar resultados educacionais mais satisfatórios do que aqueles que não desenvolvem seus potenciais afetivos em qualquer ambiente educacional (presenciais ou a distância).

As interações nas salas de aula virtuais são compostas por variadas formas de atuação e a partir de uma perspectiva ainda incipiente para a maioria dos envolvidos. As atitudes que cada participante escolhe durante as diversas atividades formarão um encadeamento de comportamentos que podem envolver grande carga afetiva, cuja maior responsabilidade para percebê-los, elaborá-los e conduzi-los será do(a) docente, na busca de um desfecho que atenda aos objetivos traçados em seu planejamento. 
As decisões cotidianamente tomadas (o que é dito e feito, de que forma isso é realizado e em que momento acontece, atravessadas pelos desejos, crenças e sentimentos) estabelecerão padrões de comportamentos que poderão qualificar (ou não) as relações entre professores e alunos, além de influenciar e afetar, em níveis diferenciados, todo o processo de ensino.

O cuidado com a atuação do professor poderá ser expandido, colaborando para uma adequada utilização das inovações tecnológicas, justificando a sistematização e a organização das potencialidades afetivas de forma dinâmica e constantemente adaptadas às demandas específicas que cada curso e/ou disciplina pode requerer.

Uma vez que se discutem e se avaliam as questões técnicas de atuação docente por um lado, fica claro que a Psicologia pode colaborar na preparação, no desenvolvimento e na avaliação dos procedimentos afetivos dos professores, considerando suas características pessoais e as que são relevantes para o exercício mais adequado de uma profissão que se caracteriza pela necessidade de uma formação contínua e dinâmica, estritamente ligada a questões de relacionamentos, liderança, planejamento, motivação e comunicação.

\section{A afetividade no contexto da educação a distância}

Os fundamentos teóricos que delineiam este artigo visam dar suporte ao processo de identificação do que afeta o professor nas suas rotinas profissionais (a partir da crescente utilização da tecnologia mediada por computadores) e a um pontual entendimento das "relações humanas", em seu aspecto afetivo.

Entende-se que a maneira de uma pessoa atuar no mundo é influenciada desde o início do seu desenvolvimento nos ambientes familiar e escolar. Tal bagagem cognitiva, psicomotora e afetiva será a base que mobilizará a atitude dos indivíduos, que atuarão dentro de uma expectativa social contextualizada e a partir das aprendizagens que as respectivas trajetórias lhes proporcionaram (Bloom, 1981).

$\mathrm{O}$ uso da tecnologia para apoio às atividades de ensino e de aprendizagem gera diferentes experiências entre alunos e professores. A mediação por computadores, em especial, tem mobilizado os participantes por apresentar possibilidades, situações e procedimentos diferenciados em relação aos recursos tradicionais disponíveis ao ensino presencial, que podem garantir economia de ordem financeira e a customização dos horários e locais de estudo dos envolvidos.

Entretanto, todas as potenciais vantagens daquelas novidades podem reduzir ou inviabilizar o sucesso de uma determinada atividade acadêmica caso os professores não conheçam suficientemente a importância dos detalhes técnicos e da forma como vão lidar (e serem afetados por eles) num ambiente virtual, bem como as possibilidades de influência dessas questões nas interações entre os participantes de suas turmas.

Os problemas que ocorrem, em sua maioria, têm origem nas relações humanas com base no conhecimento, na cultura e nas vicissitudes das experiências das pessoas. Mesmo que muitas vezes possa parecer que há um "problema na EAD”, na verdade há problemas na Educação como um todo só que com meios e recursos diferenciados. $\mathrm{O}$ que os identifica (ou não) e os caracteriza é a atuação humana. 


\subsection{Irvin Yalom: psicologia existencial e as analogias ao processo educativo:}

A afetividade impacta os processos cognitivos, as percepções e os comportamentos. Correlacionando as experiências que ocorrem nas salas de aula e nos atendimentos psicoterápicos de grupo, parece ser razoável crer que os objetivos são semelhantes.

Conforme Yalom (2006, p.19), um dos principais objetivos dos tratamentos psicoterápicos de grupos é "oferecer condições para que o indivíduo participe e trabalhe em conjunto com outras pessoas, obtenha satisfação interpessoal no contexto de relacionamentos interpessoais realistas e mutuamente satisfatórios".

Yalom (2006, p.37) afirma que a aprendizagem interpessoal representa processos únicos do ambiente de grupo, que "somente se desdobram como resultado do trabalho específico do terapeuta". Eis uma primeira e importante aproximação ao trabalho dos professores, que precisam saber flexibilizar suas intervenções e elaborar ao máximo suas afetações relacionais para oferecer o melhor ambiente para que a aprendizagem ocorra (e esses cuidados não garantem que ela irá ocorrer).

O autor destaca que a aprendizagem interpessoal é um fator terapêutico amplo e complexo. Para definir o conceito devem-se discutir dois outros conceitos: $a$ importância de relacionamentos interpessoais e o grupo como microcosmo social.

A necessidade de "fazer parte" é uma motivação poderosa e global. Adaptativamente falando, sem vínculos interpessoais profundos, positivos e recíprocos, não seria possível a sobrevivência individual ou da espécie. Sullivan (apud Yalom, 2006, p.38) diz "a personalidade é quase inteiramente um produto da interação com outros seres humanos significativos. A necessidade de se relacionar intimamente com outras pessoas é tão básica quanto qualquer necessidade biológica”.

Comparativamente, vai-se formando a capacidade de nos relacionarmos desde a participação nos grupos escolares frequentados. Um dos principais condutores desse processo são os professores por influenciar a própria forma de "ser" e "estar" no mundo.

A mediação de uma máquina e a ausência dos olhares diretos são pontos que podem dificultar a comunicação e propiciar sensações de solidão. Por isso a importância de conhecer o detalhamento dos afetos envolvidos para, mesmo que não se elaborem totalmente os problemas, que se possa ao menos dimensioná-los adequadamente.

Memórias distorcidas de interações antigas e não resolvidas influenciam na elaboração de um modelo de trabalho que estabelece padrões de apego e de reação às afetações do sujeito ao longo de sua vida, afetando a aprendizagem interpessoal.

As crenças da pessoa sobre si mesmo (como ele percebe suas relações e como tal fato influencia nos comportamentos que se seguem) evocam atitudes do outro e tendem a perpetuar-se. É no espaço do grupo que se pode trabalhar questões similares. Frequentemente um membro de um grupo altera suas percepções distorcidas após compará-las com as de outros sobre algum incidente relevante.

Então, um dos papéis importantes do "professor virtual" será o de estabelecer possibilidades para os seus alunos participarem e trabalharem com outras pessoas que possuem objetivos e desejos similares. Essa necessidade de mediar desejos e vontades poderá viabilizar a obtenção de satisfações interpessoais no contexto dos relacionamentos vinculados às respectivas realidades. 
Em ambientes virtuais essa percepção precisa ser mais efetiva, não só pela análise das produções individuais, como também pelo aumento de estratégias que harmonizem atitudes que possam trazer riscos de "ruídos na comunicação" e consequentes indisposições nos relacionamentos.

Parece justo supor que o(a) professor(a), em grande parte das situações de ensino, precisa ter uma capacidade de entendimento de suas próprias características afetivas (pois essas influenciarão os atributos anteriormente citados) para que possa facilitar seus relacionamentos interpessoais e responder às demandas diferenciadas com um repertório flexível, empático e mais adaptado aos diversos tipos de situações.

Uma das mais importantes é a elevada sensação de ansiedade que a "falta da presença" pode causar que será aliviada e modificada quando se investe na aprendizagem de uma comunicação autêntica e confiável desde o início dos contatos (Cunha et al, 2006).

Um grupo interativo livre, com poucas restrições estruturais se transformará em um microcosmo social para os participantes. Com tempo suficiente, os membros do grupo começarão a ser eles mesmos: começarão a interagir com os outros membros como interagem com pessoas em sua esfera social, criarão no grupo o mesmo universo interpessoal que sempre habitaram (Yalom, 2006, p. 46).

A EAD, ao mesmo tempo em que suscita uma liberdade espacial e temporal na resolução dos temas acadêmicos, propicia a sensação de solidão que precisa ser dimensionada e gerenciada pelo professor para que os participantes não percam o sentido dos seus esforços e possam dar continuidade adequada ao processo de ensino.

Daí a importância da aprendizagem interpessoal que, quase sempre, é relegada a um papel secundário, subjetivo, como se nada pudesse ser feito para o seu aperfeiçoamento. O cenário da sala de aula, oferece contínuas oportunidades para gerar experiências que vão afetar os comportamentos de seus integrantes.

Nesse sentido, o docente precisa estar atento para oferecer condições aos participantes que passem uma sensação autêntica de confiança, segurança e solidariedade, até para que aquelas tensões possam ser elaboradas abertamente. Além disso, deve haver um sentido de envolvimento, de comprometimento e devoluções refletidas e responsáveis sobre as questões que afetam e interessam ao grupo.

Não se pretende preparar o docente para uma análise psicológica, mas mostrar possibilidades de compreensão para intervenções que possam agregar qualidade nos seus trabalhos num contexto ainda pouco vivenciado das relações a distância.

Uma ideia fundamental da terapia de grupos que pode ser utilizada pelos professores (desde que bem elaborada e entendida por todos os atores) é o foco no "aqui-e-agora" pois, além de trazer eficácia às possibilidades de elaboração de problemas imediatos, oferece condições aos alunos de comportarem-se de forma autoreflexiva, na medida em que o professor consegue direcioná-los na busca de resoluções que corroborem o contrato pedagógico estabelecido.

O estilo interpessoal de cada membro acaba aparecendo em suas transações no grupo. As relações são tão diversas que o ciclo de eventuais transações consideradas negativas podem se repetir muitas vezes, mas ficam registradas nas ferramentas dos ambientes virtuais, o que oferece oportunidades para reflexões, entendimentos e 
proposta de soluções que só na EAD se obtém. Por um lado perde-se no contato, por outro se ganha na concretude do que está posto.

Adaptando uma precisa opinião de Yalom (2006, p.53), para que as crenças e atitudes negativas possam ser superadas, os membros da turma precisam receber devoluções claras e utilizáveis. Se o estilo de comunicação do professor for estressante demais, alguns alunos podem não conseguir processar de forma adequada o que os outros tiverem para lhes oferecer, pois poderia parecer qualquer tipo de desvalorização.

Os participantes de uma turma (alunos e professor) afetam-se continuamente. Seus relacionamentos são construídos de forma conjunta. Por isso, parece ser mais adequado reconhecer as contribuições de cada parte experimentadas no momento em que ocorrem, fortalecendo os vínculos reais que, se por um lado pode parecer diminuir o "poder do professor", por outro estabelece uma relação de confiança, de empatia e de maturidade que facilitará a resolução de problemas similares no futuro.

Os professores possuem suas problemáticas, suas próprias áreas de conflitos e de distorções interpessoais. Essas questões vivenciais certamente estarão influenciando a qualidade de suas percepções e atitudes ao longo de suas rotinas acadêmicas.

Por isso a proposta de identificar os principais atributos afetivos que envolvem as circunstâncias profissionais, na esperança de que, ao trabalhar a distância, possam compreender os limites e as novas possibilidades de suas ações para ultrapassar o cômodo silêncio sobre o que afeta as relações e elaborá-las em prol de melhores resultados educacionais.

\section{Metodologia}

A pesquisa em que se baseia este artigo, quanto a forma de abordagem, foi planejada e executada para analisar os dados de forma quantitativa e qualitativa, num recorte mais fenomenológico do que positivista (Collis, 2005, p.155).

$\mathrm{O}$ procedimento presencial consistiu em entrevistar os participantes questionando-os sobre quatro questões principais e solicitando que realizasse uma escolha forçada entre os 24 atributos afetivos apresentados numa lista em duas etapas (a primeira com a relação, em ordem alfabética, sem definições; a segunda com a mesma lista só que com as definições elaboradas de forma colaborativa em aplicações sucessivas - 2008 a 2011, e que poderiam ser alteradas caso desejassem).

As três primeiras questões levantaram as opiniões dos sujeitos sobre "a relação entre o que afeta o professor e o sucesso em suas rotinas". A segunda era sobre "quais as orientações daria a um colega que fosse trabalhar na graduação a distância pela priemira vez". A terceira queria levantar "os principais apoios que o professor naquela modalidade precisa ter". Tais questões serviam como preparação para a ordenação das listas supracitadas. A última questão versava sobre a opinião do docente a respeito da realização de uma capacitação (ou formação) afetiva antes do início de um novo curso.

Para a análise quantitativa da pesquisa foram utilizados três tipos diferentes de estatísticas: o teste $\mathrm{t}$ para amostras independentes, o teste one-way ANOVA e a correlação de Pearson (Callegari, Sidia, 2003, p.39).

Em relação aos objetivos foi exploratória, pois teve a intenção de fomentar discussões sobre uma questão pouco desenvolvida nos ambientes escolares, utilizando 
levantamentos e estudos de casos (Yin, 2001). Foi realizado um estudo de casos múltiplos com professores dos cursos de graduação à distância de sete Instituições de Ensino Superior (IES) que tiveram a experiência com turmas naquela modalidade de ensino a partir de janeiro de 2010.

A questão que orientou a pesquisa visava identificar quais os atributos afetivos daqueles são considerados mais importantes pelos próprios docentes no âmbito de suas práticas profissionais em EAD. A quantidade de 24 atributos afetivos foi delimitada a partir de trabalhos anteriores (Cunha et al, 2008).

\section{Resultados}

No período de 70 dias, no segundo trimestre de 2012, foram entrevistados 67 dos 119 docentes indicados pelas Coordenações de todas as IES que desenvolveram cursos de graduação a distância entre janeiro de 2010 e junho de 2012, realizando o máximo de estratificações na amostra (quadro 01). A duração média foi de 68 minutos.

Quadro 01 - Estratificações da amostra

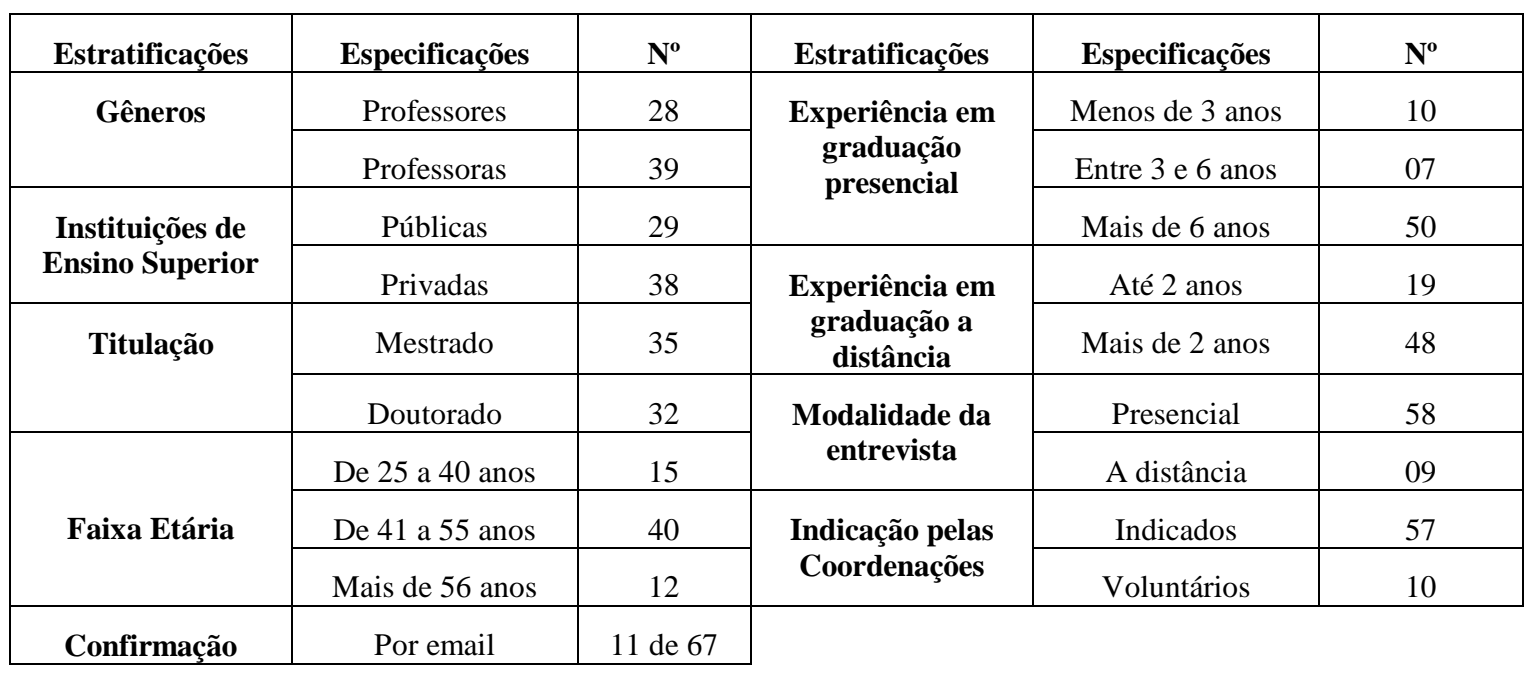

Partiu-se do pressuposto de que ao escolher em ordem prioritária entre opções reconhecidamente relacionadas às rotinas profissionais, o sujeito tende a optar por aquelas que identificam como essenciais para si e para os que exercem um papel de apoio no seu cotidiano. Essas parecem ser as mais relevantes para uma discussão sistematizada que deva ocorrer antes de iniciar um curso (de formação, por exemplo).

Dos 67 participantes, apenas cinco fizeram algum tipo de sugestão, que não modificava o sentido geral da frase alterada, mostrando que tanto os atributos selecionados para a pesquisa, quanto suas definições possuíam sentido e significado que representassem suas opiniões no que se pretendia medir (quadro 02).

Quadro 02 - Classificação Geral dos quatro primeiros e quatro últimos Atributos Afetivos em toda a amostra de participantes

\begin{tabular}{|l|l|l|l|l|l|}
\hline $\mathbf{N}^{\mathbf{0}}$ & Atributo & Média & $\mathbf{N}^{\mathbf{0}}$ & Atributo & Média \\
\hline
\end{tabular}




\begin{tabular}{|c|l|c|c|l|c|}
\hline $\mathbf{1}^{\mathbf{0}}$ & Organização (ORG) & 6,2 & $\mathbf{2 1}^{\mathbf{0}}$ & Decisão (DEC) & 15,8 \\
\hline $\mathbf{2}^{\mathbf{o}}$ & Comunicabilidade (COM) & 7,3 & $\mathbf{2 2}^{\mathbf{o}}$ & Tolerância (TOL) & 16,4 \\
\hline $\mathbf{3}^{\mathbf{0}}$ & Dedicação (DED) & 8,3 & $\mathbf{2 3}^{\mathbf{0}}$ & Imparcialidade (IMP) & 18,4 \\
\hline $\mathbf{4}^{\mathbf{o}}$ & Responsabilidade (RESP) & 9,4 & $\mathbf{2 4}^{\mathbf{0}}$ & Meticulosidade (MET) & 19,5 \\
\hline
\end{tabular}

Foram encontrados resultados estatisticamente significativos para as análises realizadas entre as médias dos três primeiros e dos três últimos atributos. Neste caso, ficou evidente que há uma clareza maior para os participantes naquilo que eles realmente pensam sobre o que pode ser mais e menos relevante para cada um na lista apresentada.

Ao analisar os resultados completos de todas as estratificações, foram identificados os principais atributos afetivos para a docência a distância. Dos oito primeiros, três deles são os mais pertinentes no contexto de qualquer subgrupo pesquisado: Organização (ORG), Comunicabilidade (COM) e Dedicação (DED).

Esses atributos podem ser utilizados tanto na realização de cursos de formação afetiva (por iniciativa das coordenações dos cursos), quanto num auto aperfeiçoamento a ser decidido em âmbito individual pelos professores interessados, haja vista que $94 \%$ da amostra considerou importante uma capacitação afetiva.

Houve, também, uma convergência na escolha dos três últimos. Uma vez que todos foram considerados relevantes pela amostra, infere-se que, ao escolher os mais importantes em suas práticas, os indivíduos (em sua maioria) mostraram as características afetivas que estão melhor introjetadas e que faziam parte de seus comportamentos cotidianos.

Assim, os atributos Tolerância (TOL), Imparcialidade (IMP) e Meticulosidade (MET) são recomendados para a seleção de novos professores (e tutores) para essa modalidade. Os quadros 03 e 04 apresentam um resumo daqueles posicionamentos.

O quadro 03 mostra as estratificações por gênero (masculino e feminino), Instituição de Ensino Superior (IES; pública e privada), titulação (mestrado e doutorado) e modalidade da entrevista (presencial e à distância).

Quadro 03: Posicionamento dos três principais atributos (primeiros e últimos).

\begin{tabular}{|c|c|c|c|c|c|c|c|c|c|}
\hline \multirow{2}{*}{$\mathbf{N}^{\mathbf{2}}$} & \multirow{2}{*}{} & \multicolumn{2}{|c|}{ Gênero } & \multicolumn{2}{c|}{ IES } & \multicolumn{2}{c|}{ Titulação } & \multicolumn{2}{c|}{ Modalidade } \\
\cline { 3 - 11 } & Amostra & Masc & Fem & Pub & Priv & Mest & Dout & Pres & AD \\
\hline $\mathbf{1}^{\mathbf{0}}$ & ORG & ORG & ORG & ORG & ORG & COM & ORG & ORG & ORG \\
\hline $\mathbf{2}^{\mathbf{o}}$ & COM & & COM & & COM & ORG & COM & COM & COM \\
\hline $\mathbf{3}^{\mathbf{o}}$ & DED & COM & DED & COM & DED & DED & DED & DED & \\
\hline $\mathbf{4}^{\mathbf{0}}$ & & DED & & DED & & & & & \\
\hline $\mathbf{5}^{\mathbf{0}}$ & & & & & & & & & DED \\
\hline $\mathbf{2 1}^{\mathbf{0}}$ & & & TOL & & $*$ & & TOL & & \\
\hline
\end{tabular}




\begin{tabular}{|c|c|c|c|c|c|c|c|c|c|}
\hline $\mathbf{2 2}^{\mathbf{0}}$ & TOL & TOL & & TOL & MET & IMP & & TOL & IMP \\
\hline $\mathbf{2 3}^{\mathbf{0}}$ & IMP & IMP & IMP & IMP & & TOL & IMP & IMP & MET \\
\hline $\mathbf{2 4}^{\mathbf{0}}$ & MET & MET & MET & MET & IMP & MET & MET & MET & TOL \\
\hline
\end{tabular}

* “Tolerância” ficou em $19^{\circ}$ lugar naquele subgrupo.

O quadro 04 mostra as estratificações da amostra dos 67 sujeitos da pesquisa por faixa etária, experiência em graduação presencial e experiência em graduação à distância.

Quadro 04: Posicionamento dos três principais atributos (primeiros e últimos).

\begin{tabular}{|c|c|c|c|c|c|c|c|c|c|}
\hline \multirow{2}{*}{$\mathbf{N}^{\mathbf{2}}$} & \multicolumn{4}{|c|}{ Faixa Etária } & \multicolumn{3}{c|}{ Experiência Presencial } & \multicolumn{2}{c|}{ Exp a distância } \\
\cline { 3 - 10 } & Amostra & $25-40$ & $41-55$ & +56 & $(-) 3$ & 3 a 6 & $(+) 6$ & Até 2 & +2 \\
\hline $\mathbf{1}^{\mathbf{0}}$ & ORG & ORG & ORG & COM & ORG & & ORG & ORG & ORG \\
\hline $\mathbf{2}^{\mathbf{o}}$ & COM & COM & COM & DED & & ORG & COM & & COM \\
\hline $\mathbf{3}^{\mathbf{0}}$ & DED & & DED & ORG & COM & & DED & & DED \\
\hline $\mathbf{4}^{\mathbf{0}}$ & & & & & DED & COM & & & \\
\hline $\mathbf{5}^{\mathbf{0}}$ & & & & & & DED & & COM & \\
\hline & & DED* & & TOL** & & & & DED* & \\
\hline $\mathbf{2 1}^{\mathbf{0}}$ & & & & & & & TOL & & TOL \\
\hline $\mathbf{2 2}^{\mathbf{0}}$ & TOL & TOL & TOL & & MET & TOL & & IMP & \\
\hline $\mathbf{2 3}^{\circ}$ & IMP & IMP & IMP & MET & TOL & IMP & IMP & TOL & IMP \\
\hline $\mathbf{2 4}^{\mathbf{0}}$ & MET & MET & MET & IMP & IMP & MET & MET & MET & MET \\
\hline
\end{tabular}

* "Dedicação" em "faixa etária, entre 25 e 40 anos" ficou em $6^{\circ}$ lugar. No subgrupo "até 02 anos em experiência a distância“ ficou em $7^{\circ}$ lugar.

** "Tolerância” em "faixa etária, com mais de 56 anos” ficou em $18^{\circ}$ lugar.

\section{Considerações finais}

A convergência de opiniões sobre o que é mais importante, aliada ao desejo demonstrado pela grande maioria da amostra em relação ao desenvolvimento de estratégias de formação afetiva, demonstra que os resultados obtidos constituem uma excelente oportunidade para trabalhar novos procedimentos e ferramentas no sentido de qualificar as afetações nos AVEA e, consequentemente, melhorar todo o processo de ensino, por sistematizar um tema que atravessa todas as rotinas, mas que não é normalmente desenvolvido.

Como trabalhos futuros, parece oportuno verificar se em outras regiões brasileiras, aplicando-se a mesma metodologia, os resultados serão convergentes para que se estabeleça uma especificação regional ou nacional das questões afetivas. 
O que parece mais significativo a partir dos resultados obtidos, de ordem prática, é elaborar um curso piloto de capacitação afetiva, com atividades presenciais e a distância que discuta os principais atributos escolhidos pela amostra atual.

Tal elaboração deve contar com uma equipe interdisciplinar que seja composta por Psicólogos, Educadores, Administradores e pessoal ligado às tecnologias informatizadas, que tenham tido a experiência de trabalho a distância para a preparação, aplicação e aperfeiçoamento de conteúdos e ferramentas específicos.

Ressalte-se que não se pretende controlar ou "engessar" procedimentos, mas sim oferecer uma metodologia que possa levantar quais os atributos afetivos mais pertinentes a determinado grupo docente, que serão definidas e priorizadas pelos profissionais envolvidos e que podem ser modificadas de acordo com as alterações necessárias a partir de avaliações constantes das rotinas e dos resultados.

\section{Referências Bibliográficas}

BLOOM, B.S. Características humanas e aprendizagem escolar: uma concepção revolucionária para o ensino. Porto Alegre: Editora Globo, 1981.

BRASIL, MINISTÉRIO DA DEFESA - EXÉRCITO BRASILEIRO. Atributos afetivos no âmbito do ensino do Exército (1998). Disponível em: http://www.dee.ensino.eb.br/legislacao/25\%20-\%20avaliacao/Port\%20012-DEP.pdf. Acesso em: 01 Jul 12.

COLLIS, J. HUSSEY, R. Pesquisa em Administração: um guia prático para alunos de graduação e pós-graduação. Porto Alegre: Bookman, 2005.

CUNHA, C.R.; BERCHT, M.; MARQUES, J. Proposta de um Modelo de Atributos para o Aprimoramento da Comunicação Afetiva para Professores que atuam na Educação a Distância. In: Anais do XIX Simpósio Brasileiro de Informática na Educação (SBIE), Fortaleza - CE, 2008.

CUNHA, C.R.; CARNEIRO, M.; HOPPE, M. Psicologias à Distância. In: Anais do XII Workshop de Informática na Educação, no âmbito do XXVI Congresso da Sociedade Brasileira de Computação, Campo Grande - MS, 2006.

YALOM, I. LESZCZ, M. Psicoterapia de Grupo: teoria e prática. Porto Alegre: ARTMED, 2006.

YIN, R.K. Estudo de caso: planejamento e métodos. Porto Alegre: Bookman, 2005.. 